\title{
Gossypiboma: a surgical menace
}

\author{
Nitin H. Shah, Aditi V. Joshi*, Vaishali N. Shah
}

Department of Obstetrics and Gynaecology, Vardann Multispeciality Hospital, Kandivali, Mumbai, Maharashtra, India

Received: 22 August 2019

Accepted: 30 September 2019

\section{*Correspondence:}

Dr. Aditi V. Joshi,

E-mail: avj111@gmail.com

Copyright: () the author(s), publisher and licensee Medip Academy. This is an open-access article distributed under the terms of the Creative Commons Attribution Non-Commercial License, which permits unrestricted non-commercial use, distribution, and reproduction in any medium, provided the original work is properly cited.

\begin{abstract}
Gossypiboma is a rare yet devastating complication. It may be a sequela to any kind of surgical procedure, however intra-abdominal surgeries are commonly implicated as the cause for this entity. In chronic cases, it may even lead to severe morbidity. We report a case of gossypiboma post vaginal hysterectomy, diagnosed and treated successfully by laparoscopy.
\end{abstract}

Keywords: Gossypiboma, Iatrogenic complication, Laparoscopy

\section{INTRODUCTION}

Gossypiboma is a foreign body reaction to a surgical item left in the body cavity. It is commonly seen following a retained gauze piece or sponge. However, other surgical items such needles, retractors, small surgical instruments may also be a cause for the same. ${ }^{1,2}$ Most patients remain asymptomatic for a prolonged duration; hence diagnosis is often delayed. Diagnosis is established by radiological imaging in the form of ultrasonography or computed tomography $(\mathrm{CT}) .^{3}$ The incidence of gossypiboma post a vaginal hysterectomy is sparse and is only occasionally reported in literature4. Every surgical team must have an adequate pre-operative and post-operative check list to avoid this complication.

\section{CASE REPORT}

55-year-old, P3L3 presented with complaints of intermittent pain in abdomen in the past 3 months. There was history of occasional episodes of fever over the last 3 months, however no other significant associated symptoms were noted. She had attained menopause 5 years back. She had three normal deliveries in the past and had undergone a vaginal hysterectomy 4 months back for a third degree uterovaginal prolapse. She was hypertensive for the last 6 years, controlled on medication. No other significant medical illness or surgical history in the past was noted.

On examination, her vital parameters were normal, P86/min, BP-140/80 mmHg. Cardiovascular and respiratory system examination was normal. On per abdomen examination, abdomen was soft with minimal suprapubic tenderness. On per speculum examination, vault was healthy with foul smelling discharge. A vaginal swab was sent for culture sensitivity. On per vaginal examination, a bogginess was felt in the fornix with tenderness on deep palpation. Her routine blood investigations were normal. An ultrasonography was done, which was suggestive of a probable pus collection in the pelvis. Vaginal swab reported a growth of " $E$. coli" and she was started on appropriate medications. A decision for laparoscopic confirmation sos drainage of the pus was considered.

A $5 \mathrm{~mm}$ primary umbilical and two $5 \mathrm{~mm}$ secondary accessory ports were inserted. On inspection, the pelvic cavity was hyperemic (Figure 1). There was a mass like structure noted at the vault, which resembled a pus collection pocket (Figure 2). The apparent mass was adherent to the surrounding area. Adhesiolysis was done, and upon further dissection, a surgical gauze piece was found (Figure 3), which was removed from the accessary 
port via a surgical glove (Figure 4). A copious saline irrigation was given.

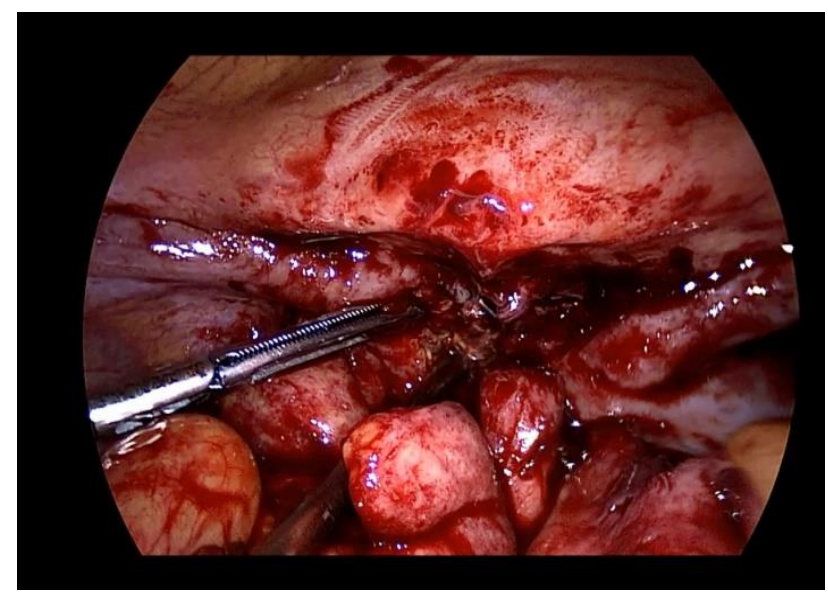

Figure 1: Illustrating the hyperemic pelvic cavity.

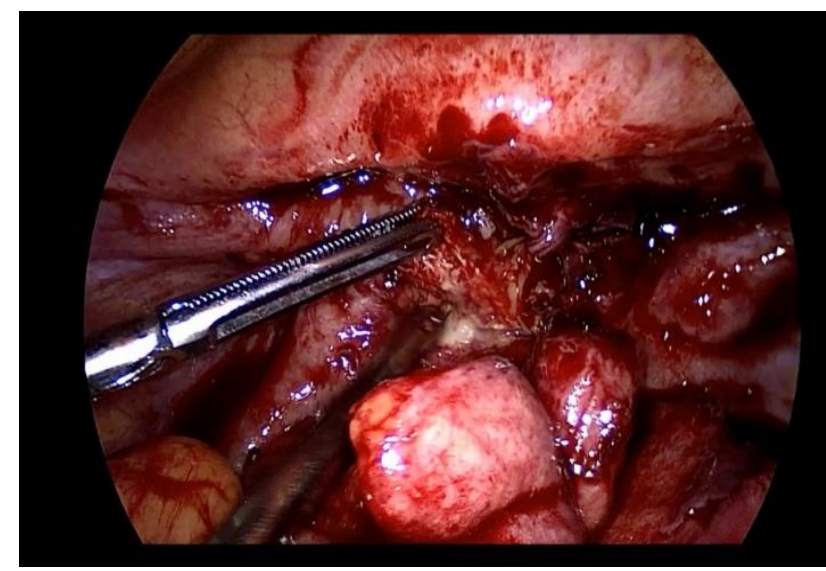

Figure 2: Depicting the probable pus collection pocket at the vault.

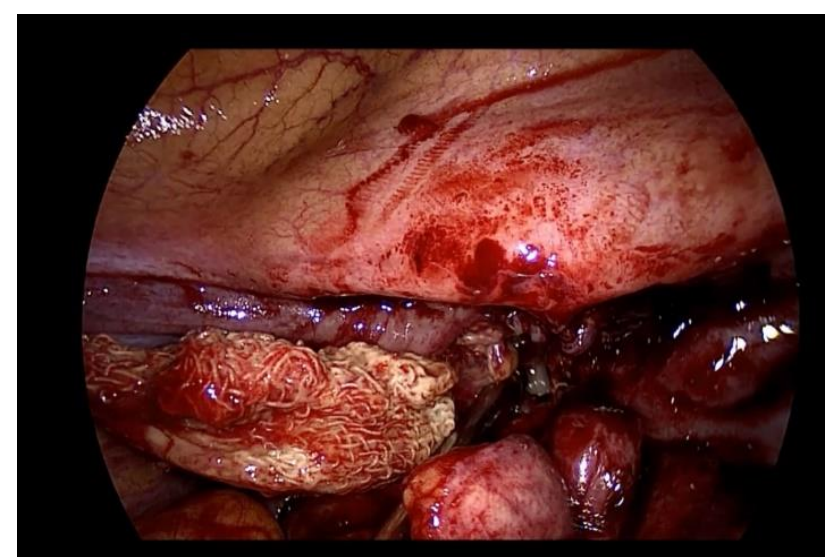

Figure 3: Illustrating a surgical gauze piece at the vault.

Her post-operative course was uneventful. Adequate antibiotic therapy was initiated and patient was discharged on the second day post procedure. A repeat ultrasound done after two weeks was normal.

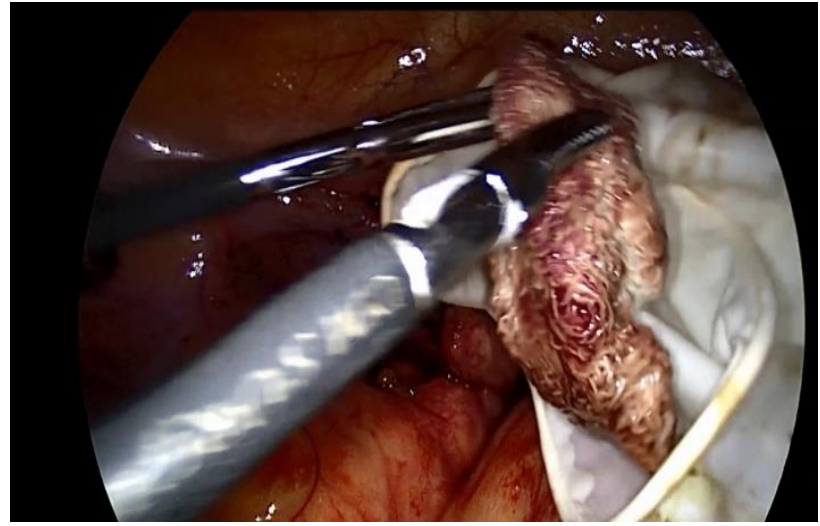

Figure 4: Retrieval of the gauze piece via a surgical glove bag.

\section{DISCUSSION}

Gossypiboma is an iatrogenic entity caused due to the retainment of a surgical swab during an operative procedure. The name is derived from a latin word "gossypium" which refers to cotton and "boma" which is Sinhalese for concealed mass after surgery. The incidence reported is sparse owing to the medicolegal implications of the same, it varies from $1 / 100$ to $1 / 3000$ in all surgical procedures. While, the incidence noted in intraabdominal procedures ranges from $1 / 1000$ to $1 / 1500$ cases. $^{5}$

There are numerous factors which can predispose to this condition, including prolonged duration of surgery, emergency surgery, excessive hemorrhage during the procedure, shortage or change of surgical personnel. Besides it has been noted that the incidence of gossypiboma is common in females and especially those with obesity. ${ }^{6}$ The presentation may vary depending upon the nature of body's reaction to the swab and may take years to manifest. In some cases, an acute inflammatory response is generated which may lead to formation of an abscess. This may manifest as fever, pain, vomiting or in some cases vaginal discharge (especially cases post hysterectomy). In others, a mass is formed due to fibrotic reaction that may present as lump in abdomen, abdominal distension or pain due to dense abdominal adhesions. ${ }^{\text {? }}$

The primary diagnosis is usually by imaging, while surgery provides the conclusive evidence. The morbidity of the procedure is lesser if the condition is diagnosed earlier. Plain X-ray may not be useful always as the radio-opaque markers may be masked or may get disintegrated over time. Ultrasonography depicts a welldefined mass with intense posterior acoustic shadowing. Computed tomography (CT) is the imaging modality of choice demonstrating the mass with well-defined capsule with a spongiform or layered appearance, in some cases. ${ }^{8}$

The management comprises of removal of the mass either by laparoscopy or laparotomy. At times the mass may migrate transmurally to the stomach or colon, in which 
case endoscopy may help to extract the mass. There are numerous studies which have demonstrated the benefit of laparoscopic surgery for gossypiboma. It enables faster recovery, shorter hospitalization and better patient compliance. ${ }^{9,10}$ Use of radiologically marked gauze or sponges is a novel method that may expediate the detection in the post-operative period. Use of embedded micro-chips that use electromagnetic or radiofrequency waves, are upcoming modalities which will hasten the diagnosis. ${ }^{11,12}$

\section{CONCLUSION}

Every surgeon and their team must be vigilant about avoiding this iatrogenic complication. A proper count of surgical equipment and swabs must be taken pre and post operatively. Laparoscopy, being a keyhole surgery proves an advantage in the surgical management of gossypiboma. Prevention is better than cure, preventing this iatrogenic complication will avoid suffering for the patient and litigation for the surgeon.

\section{Funding: No funding sources}

Conflict of interest: None declared

Ethical approval: Not required

\section{REFERENCES}

1. Manzella A, Filho PB, Albuquerque E, Farcas F, Kaecher J. Imaging gossypiboma: pictorial review. AJR. 2009;193:504-10.

2. Karahasanoglu T, Unal E, Memisoglu K, Sahinler I, Atkover G. Laparoscopic removal of a retained surgical instrument. J Laparaendosc Adv Surg Tech A. 2004;14:241-3.

3. Kiernan F, Joyce M, Byrnes CK, O'Grady H, Keane FBV, Neary P. Gossypiboma: a case report and review of the literature. Irish $\mathbf{J}$ Med Sci. 2008;177(4):389-91.

4. Oehme F, Rühle A, Stickel M, Metzger J, Gass JM. The sucked surgical sponge: Rare case of
Gossypiboma after vaginal hysterectomy. Clin Case Rep. 2019;7:753-7.

5. YX, Yu CC, Tung CF, Wu CC. Intractable duodenal ulcer caused by transmural migration of gossypiboma into the duodenum: A case report and literature review. BMC Surg. 2014;14:36.

6. Gawande AA, Studdert DM, Orav EJ, Brennan TA, Zinner MJ. Risk factors for retained instruments and sponges after surgery. The New Eng J Med. 2003;348(3):229-35.

7. Gibbs VC, Coakley FD, Reines HD. Preventable errors in the operating room: retained foreign bodies after surgery-part I. Current Prob Surg. 2007;44(5):281-337.

8. Kohli S, Singhal A, Tiwari B, Singhal S. Gossypiboma, varied presentations: A report of two cases. J Clin Imaging Sci. 2013;3:11.

9. Târcoveanu E, Dimofte G, Georgescu S. Laparoscopic retrieval of gossypibomas-short series and review of literature. Acta Chirurgica Belgica. 2011;111(6):366-9.

10. Justo JWR, Sandler P, Cavazzola LT. Retained surgical sponge mimicking GIST: laparoscopic diagnosis and removal 34 years after original surgery," J Min Access Surg. 2013;9(1):29-30.

11. Fabian CE. Electronic tagging of surgical sponges to prevent their accidental retention. Surg. 2005;137(3):298-301.

12. Macario A, Morris D, Morris S. Initial clinical evaluation of a handheld device for detecting retained surgical gauze sponges using radiofrequency identification technology. Arch Surg. 2006;141(7):659-62.

Cite this article as: Shah NH, Joshi AV, Shah VN. Gossypiboma: a surgical menace. Int J Reprod Contracept Obstet Gynecol 2019;8:4630-2. 